\title{
Occlusion of coronary vein grafts secondary to non-penetrating chest injury
}

\author{
Frank Bindi, Tom Treasure, Roy Davies
}

\begin{abstract}
Eighteen months after graft surgery to the posterior descending, circumflex, and left anterior descending coronary arteries, it seems that blunt chest trauma caused by a car accident resulted in occlusion of the grafts.
\end{abstract}

(Br Heart $\mathcal{F} 1993 ; 69: 362-363)$

Blunt chest trauma is a well established cause of cardiac injury. This was recognised by Beck in 1935 in his review of a series of 175 patients who died as a result of non-penetrating chest wounds. ${ }^{1}$ Typical presentations are with haemopericardium, myocardial changes attributable to contusion, and rupture of the atrioventricular valves (Liedke and De Muth 1973). ${ }^{2}$ Occlusion of a coronary artery has not been documented often. Sixteen cases over a 40 year period were reviewed by Vlay et al in $1980 .^{3}$ In view of an increasing population of patients who have undergone a coronary vein graft, it is important to report a case of occlusion of a saphenous vein bypass graft to the left anterior descending coronary artery, which we think, on the balance of probabilities, to have resulted from a nonpenetrating chest injury sustained in a car accident.

\section{Case report}

A 61 year old insulin dependent diabetic man presented with an eight year history of worsening angina in November 1983, by which time he was experiencing pain at rest. He had also had two previous myocardial infarctions. Investigation showed severe diffuse disease in all three vessels so he underwent grafting to the posterior descending and circumflex coronary arteries. A sequential graft was also placed to the left anterior descending coronary artery and its diagonal branch. On routine review 10 months after surgery he was well without any medication, and he reached stage III of a modified Bruce protocol without angina or ischaemic changes on the electrocardiogram.

Eighteen months after surgery, his stationary car was run into by another vehicle and he was thrown against the steering wheel (since his operation he had the habit of wedging his safety belt to take pressure off his sternotomy wound). He left his car and complained of angina-like chest pain almost immediately. He was admitted to an accident and emergency department where an initial electrocardiogram was normal. The patient experienced two further episodes of chest pain over the next five days; these were reported to be associated with ischaemic electrocardiographic changes on the monitor and new $Q$ waves on the resting electrocardiogram (unfortunately, the diagnostic traces taken at the time are not available), as well as a rise in serum hydroxybutyrate dehydrogenase to a peak of $350 \mathrm{IU} / 1$ on the fourth day. $\mathrm{He}$ was transferred back to the cardiac unit where an angiogram showed complete occlusion of the grafts to both the anterior and posterior descending coronary arteries. $\mathrm{He}$ continued to have unstable angina, and even with treatment, had pain on exercise.

The close association in time between the road accident and the return of symptoms, coupled with the subsequent electrocardiographic changes and rise in enzyme concentration, strongly suggests that the blunt trauma resulted in the acute occlusion of his important left anterior descending graft.

\section{Discussion}

The incidence of non-penetraing chest trauma with subsequent cardiac injury has increased with the rising popularity of car travel. Vlay et al showed that automobile accidents were implicated in $58 \%$ of 16 cases of native coronary artery occlusion; the most common mechanism was, as in our case, steering wheel trauma. ${ }^{4}$ (Madoff 1972). Assault was involved in $19 \%$ of patients. The remaining $31 \%$ occurred as a result of sporting and industrial accidents.

Laboratory experiments with animals have shown a high correlation between severe trauma and myocardial contusion; ${ }^{5}$ however, violent injury does not seem to be necessary to cause discrete coronary artery occlusion, and the absence of rib or sternal fractures is a common feature. ${ }^{6}$

The actual mechanism of coronary artery occlusion after trauma is probably multifactorial. Possible aetiologies include atheroma with haemorrhage into a plaque, spasm within normal coronary arteries, coronary embolisation, and coronary thrombosis. The existence of disease within the vessel may be an important factor. ${ }^{7}$

Another important consideration is the relation between the vessel and the direction of trauma. Levy in 1939 thought that vessels lying superficially on the muscle wall were particularly prone to damage, especially if dis- 
eased. ${ }^{8}$ Anatomically, the left anterior descending coronary artery ought to be at greatest risk. This was confirmed by Vlay et al who showed occlusion rates of $63 \%$ in the left anterior descending, $25 \%$ in the right coronary, and $12 \%$ in the left circumflex arteries. ${ }^{3}$ Our patient had grafts on the left anterior descending and the right coronary arteries.

The difficulty in cases such as ours lies in establishing the causal relation between trauma and occlusion of the grafts. It is generally accepted that the combination of sudden start of symptoms shortly after trauma and electrocardiographic changes makes the correlation extremely likely. ${ }^{6}$ The presence of positive angiographic changes in our patient further reinforces this argument. However, Boas, as early as $1939,{ }^{6}$ and Sigmund et al, as late as $1990,{ }^{9}$ reported cases in which there was a lag period of days between injury and subsequent thrombotic occlusion of the vessel. This would seem to correlate well with our case where pain with ischaemic electrocardiographic changes appeared four days after the accident.

The medicolegal implications are an obvious cause for concern; this case led to civil action by the patient against the driver of the other vehicle. This was pursued as far as the court but once the evidence was presented, after five years had elapsed, as is often the case with legal matters, the defendents agreed to settle out of court.

As far as the patient's treatment was concerned, a further attempt at coronary surgery was not considered advisable and the patient continued on medical management.

1 Beck CS. Contusions of the heart. FAMA 1935;104: 109-14.

2 Liedke AJ, De Muth WE jr. Non-penetrating cardiac

injuries: collective review. Am Heart $\mathcal{f} 1973 ; 86: 687-97$.
3 Vlay SC, Blumenthal DS, Shoback D, Fehir K, Bulkley BH. Delayed acute myocardial infarction after blunt BH. Delayed acute myocardial infarction after blunt
chest trauma in a young woman. Am Heart $\mathcal{f} 1980 ; 100$ : chest traum

4 Madoff IM, Desforges G. Cardiac injuries due to nonpenetrating thoracic trauma. Am Thor Surg 1972;14: 504-12.

5 Bright EF, Beck CS. Non-penetrating wounds of the heart. Am Heart $\mathcal{f}$ 1934;10:293-321.

6 Boas ET. Angina pectoris and myocardial infarction from trauma or unusual effort. $\mathcal{F A M A}$ 1939;112:1887-92.

7 Greene HL, Reid PR, Sheaffer AH. The repetitive ventricular response: a predictor of sudden death. $N$ Engl $f$ Med 1978;299:729-34.

8 Levy $\mathrm{H}$. Traumatic coronary thrombosis with myocardial infarction. Arch Intern Med 1949;84:261-76.

9 Sigmund $M$, Nase-Huppmeier S, Uebis R, Hanrath $P$. Emergency PTCA for coronary artery occlusion after blunt chest trauma. Am Heart $\mathcal{f}$ 1990;119:1408-10. 